\title{
The response of high-purity germanium detectors to X-rays with energy in the region of the Ge K-absorption edge
}

\author{
J.M.F. dos Santos*, C.M.B. Monteiro \\ Physics Department, University of Coimbra, P-3004-516 Coimbra, Portugal
}

Received 10 August 2000; received in revised form 3 January 2001; accepted 27 January 2001

\begin{abstract}
The response of a high-purity germanium detector to X-rays in the $8-15-\mathrm{keV}$ energy region has been investigated. The $w$-value and energy resolution dependencies on the X-ray energy have been studied. No abrupt variation of $w$ is observed at the germanium $\mathrm{K}$ absorption-edge $(11.104-\mathrm{keV})$. The detector energy resolution follows a characteristic linear dependence on $E_{x}^{-1 / 2}$ over the whole energy range. Both features are consistent with the absence of intrinsic nonlinearity effects in germanium at the K-edge. (C) 2001 Elsevier Science Ltd. All rights reserved.
\end{abstract}

Keywords: HPGe; Energy linearity; K-edge

\section{Introduction}

High-purity germanium detectors (HPGe) are often used in X-ray spectrometry for energies down to a few $\mathrm{keV}$. Knowledge of detailed detector performance, particularly energy resolution and linearity, is necessary for accurate measurements. Through the years both quantities have been investigated as instrumental responses have evolved to higher levels of precision. An accurate energy calibration of a radiation detector, especially at the lower end of its operating range, requires a detailed knowledge of its energy response.

Discontinuities in the response of gaseous detectors to $\mathrm{X}$-rays have been observed at energies around the absorption edges of the detection medium (Lamb et al., 1987; Jahoda and McCammon, 1988; Santos et al., 1991; dos Santos et al., 1993; Tsunemi et al., 1993; dos Santos et al., 1994; Budtz-Jorgensen et al., 1995; Dias et al., 1997). An abrupt increase of the $w$-value, the average energy to originate an electron/ion pair, occurs at these energies, resulting in non-linearity effects in the detector response. Between edges, the $w$-value decreases slightly, approaching a constant value for X-ray energies

\footnotetext{
*Corresponding author. Fax: + 351-239-829158.

E-mail address: jmf@gian.fis.uc.pt (J.M.F. dos Santos).
}

well above the most energetic absorption-edge. An explanation for these discontinuities is supported by Monte Carlo simulations (Santos et al., 1991; Dias et al., 1997): the efficiency for converting the absorbed X-ray energy into ionisation is lower for atomic sub-shells with higher binding energies. When a new photo-ionisation channel becomes energetically accessible, the subsequent de-excitation cascade of the photo-ionised atom results in a larger number of electron vacancies in the outermost sub-shells. A measurable amount of the absorbed energy is expended in establishing the ground state of the ion and the additional vacancies. At still higher photon energies, the energy dissipated in establishing the cascade vacancies is a smaller fraction of the total energy transferred to the photoelectrons, and an approximate constant $w$-value is restored. However, although the continuous variation of $w$ with X-ray energy cannot be neglected, the effect of this variation on the detector response is small and good energy linearity is held, except at the absorption edges, where an abrupt decrease in the detector pulse amplitude occurs (dos Santos et al., 1993).

Experimental and Monte Carlo simulation studies implemented for silicon detectors have revealed the same trend discussed above (Fraser et al., 1994; Owens et al., $1996)$, and an energy discontinuity of $\sim 0.2 \%(\sim 3.6 \mathrm{eV})$ 
was obtained at the Si K-edge. However, Torii et al. (1995) have measured a discontinuity of $1.5 \pm 2.6 \mathrm{eV}$ and concluded in their study, that there is no intrinsic nonlinearity effect in silicon at the K-edge.

Discontinuities at the K-edge in germanium are not as well documented as those for Si. In a former study (dos Santos et al., 2000) we have investigated the energy linearity of an HPGe detector in the photon energy region of $8-15 \mathrm{keV}$. X-ray energies below and above the germanium K-edge were produced by exciting $\mathrm{K}$-fluorescence in selected target elements and the detector pulse amplitude was investigated as a function of the X-ray energy. The results were plotted as a function of the energy and fitted by straight lines below and above the K-edge. Extrapolating each straight line to the energy corresponding to the germanium K-edge threshold $(11.104 \mathrm{keV})$, a discontinuity of $3 \pm 4 \mathrm{eV}$ was measured. This hypothesis was compared against a single line fitting to all data points. A $\chi^{2}$ analysis for the two hypotheses suggests the data to be more consistent with the two-lines fitting, supportive of a possible discontinuity. However, the discontinuity is less than $0.1 \%$.

\section{Present investigation}

To better clarify the existence or absence of an intrinsic discontinuity at the Ge K-edge, we performed further analysis of the detector response, investigating the behaviour of the $w$-value and the detector energy resolution as a function of the X-ray energy.
The detector used was a planar Ortec GLP HPGe, with an $8 \mathrm{~cm}^{2} \times 1-\mathrm{cm}$ volume and a thin front contact of less than $0.3 \mu \mathrm{m}$. Throughout the experiment, the detector was biased at $-1500 \mathrm{~V}$. The built-in preamplifier pulses were fed through an Ortec 575 A amplifier, using shaping times of $3 \mu \mathrm{s}$, to a 4096-channel 'The Nucleus' MCA. The counting rate in the detector was maintained below $100 \mathrm{cps}$ in all cases, a rate sufficiently low to neglect any dead time and pile-up effects. By maintaining a low counting rate, any rate effects due to the abrupt increase in the absorption efficiency at the K-edge were minimised.

The required X-ray energies were generated by exciting $\mathrm{K}$-fluorescence lines in selected target elements using a ${ }^{241} \mathrm{Am}$ source, in a $90^{\circ}$ configuration, as described in dos Santos et al. (2000). A 10-mm-diameter collimator positioned over the $2.54-\mathrm{cm}$ detector entrance window limited the field of view to avoid any edge effects and to minimise any effect due to possible variations in the excitation-detection geometry. Peak centroid positions and full-widths-at-half-maximum were determined by fitting the measured pulse-height distributions to gaussians superimposed on a linear background, using the Grid Least-Squares fit method (Bevington, 1969).

\section{Results}

System stability was determined by monitoring the centroid-peak position of the characteristic radiation of zinc and strontium throughout the data acquisition period. During this period, several pulse-height

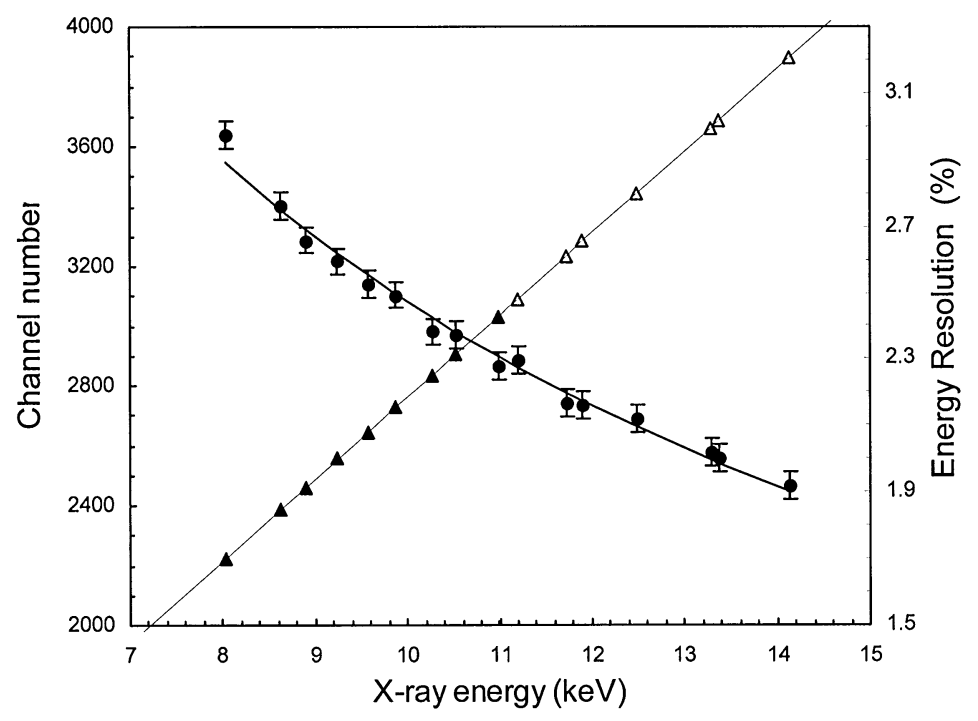

Fig. 1. Peak centroid $(\boldsymbol{\Delta}, \triangle)$ and energy resolution $(\mathbf{\bullet})$ as a function of the X-ray energy, $E_{x}$. The solid straight lines represent the least-squares fits of each set of data below $(\mathbf{\Lambda})$ and above $(\triangle)$ the germanium K-edge. The solid curved line represents the least squares fit to $E_{x}^{-1 / 2}$. 


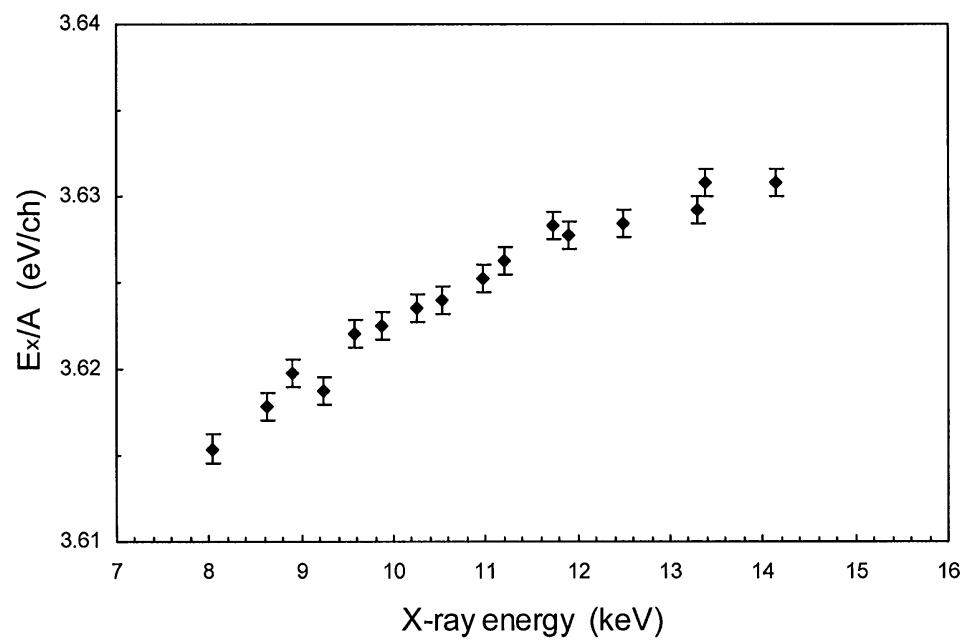

Fig. 2. X-ray energy-to-centroid channel ratio, $E_{x} / A$, as a function of X-ray energy.

distributions of $\mathrm{Zn}$ and $\mathrm{Sr}$ have been obtained, at constant time intervals. System stability was found to be within $0.02 \%$. The errors in the measured centroid-peak position as well as in the energy resolution were estimated using these different pulse-height distributions. Additionally, the uncertainties introduced by the peak fit algorithm have also been considered by determining the effect of different regions-of-interest on the centroid-peak position and energy resolution. In this manner, the errors in the centroid position and in the energy resolution were determined to be less than 0.4 channels and $0.05 \%$, respectively.

In Fig. 1 the peak centroids and energy resolutions are plotted as a function of the energy, together with the least-squares fit of each set of data, below and above the germanium K-edge threshold, to a straight line, resulting in a discontinuity of $3 \pm 4 \mathrm{eV}$.

Assuming a detector gain $G$, the centroid channel number, $A$, and the average number of primary electrons, $N$, produced by the X-rays with energy, $E_{x}$, are related by

$A=G N$.

The $w$-value, the average energy to produce a pair of charge carriers, is given by

$w=\frac{E_{x}}{N}=G \frac{E_{x}}{A}$.

In Fig. 2 we present the $E_{x} / A$ ratio as a function of the X-ray energy. As seen, no abrupt variation, characteristic of a discontinuity in the detector response, is observed. This behaviour is consistent with the absence of an intrinsic non-linearity effect in $\mathrm{Ge}$ at the $\mathrm{K}$-absorption edge. The observed slight increase with Xray energy departs from the characteristic behaviour of an ideal detector (Fraser et al., 1994; Dias et al., 1997)



Fig. 3. Energy resolution as a function of $E_{x}^{-1 / 2}$. The solid line represents the least squares fit to $E_{x}^{-1 / 2}$.

and can be attributed to the loss of charge carriers in the lattice imperfections due to trapping. This effect can be significant in a large volume detector with relatively large charge drift distances (Knoll, 2000). However, the increase of $w$ that may be observed in real detectors does not invalidate measured results of their response to Xrays (e.g. Dias et al., 1997). Independent of the increase or decrease obtained in the $w$ behaviour, a non-linearity in a detector response is associated with an abrupt variation in the measured $w$-value. As discussed in another paper (dos Santos et al., 1993), although the continuous variation of $w$ with $E_{x}$ cannot be neglected, its effect on the detector response linearity is negligible.

Figs. 1 and 3 depict the detector energy resolution obtained for the given experimental conditions, as a function of the radiation line energy. As shown in Fig. 3, the detector energy resolution follows the characteristic 
energy dependence with $E_{x}^{-1 / 2}$ over the whole energy range. The results of the $\chi^{2}$ analysis for a single line fitting to all data points is similar to the one for a twoline fitting to each set of data, above and below the Ge $\mathrm{K}$-edge, which is also consistent with the absence of an intrinsic non-linearity effect in $\mathrm{Ge}$ at the $\mathrm{K}$-absorption edge.

\section{Conclusions}

As our results indicate, the energy expenditure for the initially photo-ionised atom and the interactions of photoelectrons in a solid crystalline detector is obviously more complicated than in a gaseous medium. Collective effects, which are beyond the scope of this paper, may well dominate the $\mathrm{X}$-ray energy absorption process. The behaviour of the $w$-value and detector energy resolution are both consistent with the absence of intrinsic nonlinearity effects in germanium at the K-edge.

\section{Acknowledgements}

The work was carried out in the laboratory of the Atomic and Nuclear Instrumentation Group of the Instrumentation Center (Unit 217/94) of Departamento de Física, Universidade de Coimbra. Support is acknowledged from project PRAXIS/3/3.1/CTAE/ 1920/95. We thank R.E. Morgado (LANL, USA) for helpful discussions.

\section{References}

Bevington, P.R., 1969. Data Reduction and Error Analysis for the Physical Sciences. McGraw-Hill, New York, pp. 208-214. Budtz-Jorgensen, C., Olesen, C., Schnopper, H.W., Lederer, T., Scholze, F., Ulm, G., 1995. The response functions of the HEPC/LEPC detector system measured at the Xe L edge region. Nucl. Instr. and Meth. A 367, 83-87.

Dias, T.H.V.T., Dos Santos, J.M.F., Rachinhas, P.J.B.M., Santos, F.P., Conde, C.A.N., Stauffer, A.D., 1997.
Full-energy absorption of X-ray energies near the Xe L-and K-photoionization thresholds in xenon gas detectors: Simulation and experimental results. J. Appl. Phys. 82 (6), 2742-2753.

Dos Santos, J.M.F., Conde, C.A.N., Bento, A.C.S.S.M., 1993. The energy linearity of gaseous xenon radiation detectors for $\mathrm{X}$-rays with energies between 2 and $60 \mathrm{keV}$ : experimental results. Nucl. Instr. and Meth. A 324, 611-613.

Dos Santos, J.M.F., Morgado, R.E., Tavora, L.M.N., Conde, C.A.N., 1994. The energy nonlinearity of gaseous proportional scintillation counters at the $\mathrm{K}$-absorption edge in xenon. Nucl. Instr. and Meth. A 350, 216-220.

Dos Santos, J.M.F., Monteiro, C.M.B., Morgado, R.E., Conde, C.A.N., 2000. Energy Linearity of High-Purity Germanium Detectors in the Region of the Ge K-absorption edge: experimental Results. Appl. Rad. Isot. 53, 739-743.

Fraser, G.W., Abbey, A.F., Holland, A., McCarthy, K., Owens, A., Wells, A., 1994. The X-ray energy response of silicon Part A: Theory. Nucl. Instr. and Meth. A 350, 368-378.

Jahoda, K., McCammon, D., 1988. Proportional counters as low-energy photon detectors. Nucl. Instr. and Meth. A 272, $800-813$.

Knoll, G.F., 2000, Radiation Detection and Measurements. 3rd Edition, Wiley, New York, pp. 416-418.

Lamb, P., Manzo, G., Re, S., Boella, G., Villa, G., Andresen, R., Sims, M.R., Clark, G.F., 1987. The gas scintillation proportional counter in the spacelab environment: In-flight performance and post-flight calibration. Astrophys. Space Sci. A 136, 369-378.

Owens, A., Fraser, G.W., Abbey, A.F., Holland, A., McCarthy, K., Keay, A., Wells, A., 1996. The X-ray energy response of silicon (B): Measurements. Nucl. Instr. and Meth. A 382, 503-510.

Santos, F.P., Dias, T.V.H.T., Stauffer, A.D., Conde, C.A.N., 1991. Variation of energy linearity and $w$ value in gaseous xenon radiation detectors for X-rays in the 0.1 to $25 \mathrm{keV}$ energy range: a Monte Carlo simulation study. Nucl. Instr. and Meth. A 307, 347-352.

Torii, K., Tsunemi, H., Miyata, E., Hayashida, K., 1995. Some characteristics of a solid state detector in the soft X-ray region. Nucl. Instr. and Meth. A 361, 364-371.

Tsunemi, H., Hayashida, K., Torii, K., Tamura, K., Miyata, E., Murakami, H., Ueno, S., 1993. Nonlinearity at the Kabsorption-edge in the Xe filled gas proportional counter. Nucl. Instr. and Meth. A 336, 301-303. 